\title{
Microstructure Investigations of $\mathrm{Yb}$ - and $\mathrm{Bi}$-doped $\mathrm{Mg}_{2} \mathrm{Si}$ Prepared from Metal Hydrides for Thermoelectric Applications
}

Oliver Janka ${ }^{\mathrm{a}, \mathrm{d}}$, Julia Zaikina ${ }^{\mathrm{a}}$, Sabah Bux ${ }^{\mathrm{b}}$, Hosna Tabatabaifar ${ }^{\mathrm{a}}$, Hao Yang ${ }^{\mathrm{c}}$, Nigel D. Browning $^{\mathrm{c}}$, Susan M. Kauzlarich ${ }^{*}$ a

a Department of Chemistry, University of California, Davis, One Shields Avenue, Davis, CA 95616, USA

b Jet Propulsion Laboratory/California Institute of Technology, Thermal Energy Conversion Technologies Group, Jet Propulsion Laboratory, Pasadena, CA 91125, USA.

c Chemical and Materials Sciences Division, Pacific Northwest National Laboratory, Richland, WA 99352, USA.

d Current address: Institut für Anorganische und Analytische Chemie, Westfälische Wilhelms-Universität, Corrensstrasse 28/30, 48161 Münster, Germany. 


\begin{abstract}
Within the field of thermoelectric materials for energy conversion magnesium silicide, $\mathrm{Mg}_{2} \mathrm{Si}$, is an outstanding candidate due to its low density, abundant constituents and low toxicity. However electronic and thermal tuning of the material is a required necessity to improve its Figure of Merit, $z T$. Doping of $\mathrm{Yb}$ via reactive $\mathrm{YbH}_{2}$ into the structure is performed with the goal of reducing the thermal conductivity. Hydrogen is released as a byproduct at high temperature allowing for facile incorporation of $\mathrm{Yb}$ into the structure. We report on the properties of $\mathrm{Yb}$ - and $\mathrm{Bi}$-doped $\mathrm{Mg}_{2} \mathrm{Si}$ prepared with $\mathrm{MgH}_{2}$ and $\mathrm{YbH}_{2}$ with the focus on the synthetic conditions, and samples' microstructure, investigated by various electron microscopy techniques. $\mathrm{Yb}$ is found in the form of both $\mathrm{Yb}_{3} \mathrm{Si}_{5}$ inclusions and $\mathrm{Yb}$ dopant segregated at the grain boundary substituting for $\mathrm{Mg}$. The addition of 1 at.\% $\mathrm{Yb}$ concentration reduced the thermal conductivity, providing a value of $30 \mathrm{~mW} / \mathrm{cm} \cdot \mathrm{K}$ at $800 \mathrm{~K}$. In order to adjust carrier concentration, the sample is additionally doped with Bi. The impact of the microstructure on the transport properties of the obtained material is studied. Idealy, the reduction of the thermal conductivity is achieved by doping with $\mathrm{Yb}$ and the electronic transport is adjusted by doping with $\mathrm{Bi}$. Large grain microstructure facilitates the electronic transport. However, the synthetic conditions that provide the optimized microstructure for electrical transport do not facilitate the additional $\mathrm{Yb}$ dopant incorporation. Therefore, the $\mathrm{Yb}$ and Bi containing sample with the optimized microstructure provides a $z T=0.46$ at $800 \mathrm{~K}$.
\end{abstract}

\title{
KEYWORDS
}

Magnesium silicide, Thermoelectric Materials, Ytterbium, Bismuth 


\section{Introduction}

First studies on the thermoelectric properties of Zintl phases of the composition $\operatorname{Mg}_{2} X(X=$ $\mathrm{Si}, \mathrm{Ge}, \mathrm{Sn})$ and their solid solutions were made about 50 years ago [1, 2]. These investigations showed their promising potential for thermoelectric applications, because of the large Seebeck coefficients, low electrical resistivity, along with thermal stability, environmentally friendly elements with relative abundance and low density. Research on these materials has recently significantly increased because they are considered to be high-performance thermoelectric materials between $500 \mathrm{~K}$ and $900 \mathrm{~K}$, which can convert wasted heat produced by functioning automobile exhaust into useful electricity [1-4].

$\mathrm{Mg}_{2} \mathrm{Si}$ needs modifications to obtain good thermoelectric properties, such as reduction of the lattice conductivity and increase in carrier mobility. In order to decrease the thermal conductivity several ideas have been discussed in literature. One is to replace the $\mathrm{Mg}^{2+}$ in the crystal structure of $\mathrm{Mg}_{2} \mathrm{Si}$ by a heavier element. This will introduce disorder into the crystal lattice affecting scattering of heat carrying phonons, thus reducing thermal conductivity. Meng et al. have shown that this is possible with yttrium, Y [5], and Todaka et al. published thermoelectric data on sodium, $\mathrm{Na}$, -doped $\mathrm{Mg}_{2} \mathrm{Si}$ [6]. In these examples the divalent $\mathrm{Mg}$ cation is replaced by either a trivalent or monovalent cation, which leads to defects in the crystal structure and consequently affecting the carrier concentration. Therefore it is not possible to isolate the effect of the substituting element. To avoid this problem, Zhao and coworkers showed that the substitution of $\mathrm{Mg}^{2+}$ with isovalent $\mathrm{Ca}^{2+}$ is possible according to $\mathrm{Mg}_{2-x} \mathrm{Ca}_{x} \mathrm{Si}$ with $x$ up to 0.1 [7]. Since the thermal conductivity is related to the scattering of the phonons by crystal lattice vibrations, heavier elements should be more effective scattering centers and therefore reduce the phonon mean free path more efficiently.

Two other approaches to lower thermal conductivity are to make nanocrystalline $\mathrm{Mg}_{2} \mathrm{Si}$ [8] or to incorporate nanoparticles to form composite materials [10]. Mingo and co-workers have shown with calculations on thermal conductivity that metal silicide nanoparticles have a significant influence when doped into $\mathrm{Si}_{1-x} \mathrm{Ge}_{x}$ alloys [9]. Silicon nanoparticles for example have been experimentally shown to affect the thermal conductivity by reducing the phonon mean free path in $\mathrm{Mg}_{2} \mathrm{Si}$ [10]. This has been also found to be true for $\mathrm{Mg}_{2} \mathrm{Si}_{x} \mathrm{Ge}_{y} \mathrm{Sn}_{1-x-y}$ [11]

In addition to reducing the thermal conductivity in $\mathrm{Mg}_{2} \mathrm{Si}$, the electrical transport also needs to be adjusted. The optimization of charge carrier concentration can be achieved by doping $\mathrm{Mg}_{2} \mathrm{Si}$ with group $15^{\text {th }}$ elements, such as $\mathrm{Sb}$ or $\mathrm{Bi}$ [12-15]. These dopants have a combined effect on the material, since doping with these heavy elements increases the scattering of heat carrying phonons due to point defect scattering and at the same time add 
excess electrons, which affects the carrier concentration. For plain $\mathrm{Mg}_{2} \mathrm{Si}$, Bi doping has shown the best results to date with zT $\sim 0.7$ at $775 \mathrm{~K}$ [15-19].

The reactive spark plasma sintering (SPS) of the magnesium hydride and doped silicon was shown to be facile route for the one-step preparation and densification of $\mathrm{Mg}_{2} \mathrm{Si}$-based materials [10]. The process facilitates preparation of the oxide-free magnesium silicide products with high density $[8,10,20]$. Herein, we present the effect of $\mathrm{Yb}$ and Bi doping into $\mathrm{Mg}_{2} \mathrm{Si}$ via a $\mathrm{MgH}_{2}$ route and the microstructure study of as a function of the sintering temperature in order to provide a better understanding of the dopant and microstructureproperty relationship. Addition of $\mathrm{YbH}_{2}$ to the synthetic route is used to further reduce thermal conductivity. There are two results of the addition of $\mathrm{YbH}_{2}$. The first is the isovalent substitution of $\mathrm{Mg}^{2+}$ by heavier $\mathrm{Yb}^{2+}$ and the second is the formation of a $\mathrm{Yb}$-containing precipitate in the $\mathrm{Mg}_{2} \mathrm{Si}$ matrix. Bi dopant is employed to control the transport properties. The microstructure is studied as a function of ball milling and sintering temperature. The resulting composite phase is characterized and the impact of dopant and microstructure on the thermoelectric properties is discussed.

\section{Results and Discussion}

\subsection{Synthesis}

Thermogravimetry / differential scanning calorimetry (TG/DSC) investigations indicated that this reaction proceeds in the manner described according to reaction (1). (See Supporting Information)

$$
2 \mathrm{MgH}_{2}+\mathrm{Si} \rightarrow \mathrm{Mg}_{2} \mathrm{Si}+2 \mathrm{H}_{2}
$$

The results are similar to those previously reported [10] and in good agreement with the reported decomposition temperatures of $\mathrm{MgH}_{2}: 652 \mathrm{~K}$ in Spark Plasma Sintering (SPS) process and $667 \mathrm{~K}$ in DSC [20, 21]. The endothermic peak with its onset at $671 \mathrm{~K}$ is close to $680 \mathrm{~K}$ determined in the detailed studies regarding the reaction kinetics in the $\mathrm{Mg}-\mathrm{Si}-\mathrm{H}$ system [22]. Similar to previous reports [10][22], the mass loss is lower than the expected loss of hydrogen (5 wt- $\%$ ) based on the reaction equation. Reaction kinetics [22] show that activation rates are strongly correlated with particle size. The reaction does not take place in the the ball milling step [22]; in contrast to the mechanical alloying of Si with magnesium metal to form $\mathrm{Mg}_{2} \mathrm{Si}[17,20]$.

The ball milled powders of $\mathrm{MgH}_{2}, \mathrm{Si}$ and the respective $\mathrm{Bi}$ and/or $\mathrm{YbH}_{2}$ dopants were sintered and reacted in a single step using the SPS technique. The ball-mill time varies and is 
denoted as short for two sets of 30 minutes or long for 9 hours of ball-milling in 15 minute on/off intervals (see experimental and Table 1). The long ball-milling time is slightly shorter than that previously reported $[8,10]$, but was deemed sufficient based on the resulting powder $\mathrm{X}$-ray diffraction of the SPS powder. The short ball-milling was determined by powder X-ray diffraction to also be sufficient for producing nearly phase pure $\mathrm{Mg}_{2} \mathrm{Si}$. The sintering temperature was investigated as well and Table 1 provides the details for each sample. The maximum temperature of $1023 \mathrm{~K}$ was chosen as the highest possible to apply without significant loss of $\mathrm{Mg}$.

Table 1: Synthetic conditions used to obtain the samples discussed herein.

\begin{tabular}{llclcc}
\hline ID & Yb (at.-\%) & Bi (at.-\%) & ball millingt & temperature(K) & density, $\%$ \\
\hline I & 1.0 & - & short & 973 & $98 \%$ \\
II & 0.5 & - & short & 973 & $97 \%$ \\
III & 0.2 & - & short & 973 & $100 \%$ \\
IV & 1.0 & 1.0 & long & 773 & $98 \%$ \\
V & 1.0 & 1.0 & long & 923 & $95 \%$ \\
VI & 1.0 & 1.0 & long & 1023 & $99 \%$ \\
VII & - & 1.0 & short & 973 & $98 \%$ \\
VIII & 0.2 & 1.0 & short & 973 & $97 \%$ \\
\hline
\end{tabular}

\$ short $=$ two sets of 30 minutes; long $=9$ hours of 15 minute on/off intervals

\subsection{Lowering thermal conductivity with $\mathrm{YbH}_{2}$ - proof of concept and optimization}

In order to investigate the effect of $\mathrm{Yb}$, either as a dopant or alloy, on the thermoelectric properties, three samples with different $\mathrm{Yb}$ dopant concentrations $(0.2 \%, 0.5 \%$ and $1.0 \%$ (IIII)) were prepared via a short ball-milling approach (Table 1). Short ball-milling was explored as it has been shown that nanopowders of Si can be problematic for forming dense pellets [20]. Stoichiometric amounts of $\mathrm{MgH}_{2}, \mathrm{YbH}_{2}$ and $\mathrm{Si}$ along with the dopants were ballmilled and loaded into graphite dies. The reactants were handled as described in the experimental section (vide infra) and sintered in the SPS as described above. The obtained samples were investigated by powder X-ray diffraction and showed $\mathrm{Mg}_{2} \mathrm{Si}$ and a small amount of Si. No peak broadening was observed, indicating that the material particle size after SPS is $\geq 100 \mu \mathrm{m}$. $\mathrm{Yb}_{3} \mathrm{Si}_{5}$ was observed in the samples with $1 \%$ of $\mathrm{Yb}$ prepared via long ball-milling and in the sample VIII prepared via short ball-milling. No other ytterbium silicides were identified. Within the error of the measurement and the absence of an internal standard, no change of the lattice parameters was observed. The X-ray powder diffraction patterns of all samples are provided in the Supporting Information (Fig. S2-S9). 
To obtain more information about the elemental distribution within the pressed pellets, a $1 \% \mathrm{Yb}$ containing $\mathrm{Mg}_{2} \mathrm{Si}$ sample (I) was investigated using electron microprobe analysis. $\mathrm{X}$ ray mapping was used to display the elemental distribution within an area of $200 \times 200 \mu \mathrm{m}^{2}$ (Fig. 1). As seen from the X-ray maps, $\mathrm{Mg}$ poor areas contain excess $\mathrm{Si}$. Electron microprobe analyses showed the expected composition of the matrix, the determined doping levels were close to the experimentally used concentrations. For $\mathrm{Yb}$, low counts can be seen across the whole sample, which gives rise to the assumption that $\mathrm{Yb}^{2+}$ was alloyed into $\mathrm{Mg}_{2} \mathrm{Si}$, along with bright spots, which can be attributed to the $\mathrm{Yb}_{3} \mathrm{Si}_{5}$ phase identified by powder X-ray diffraction.

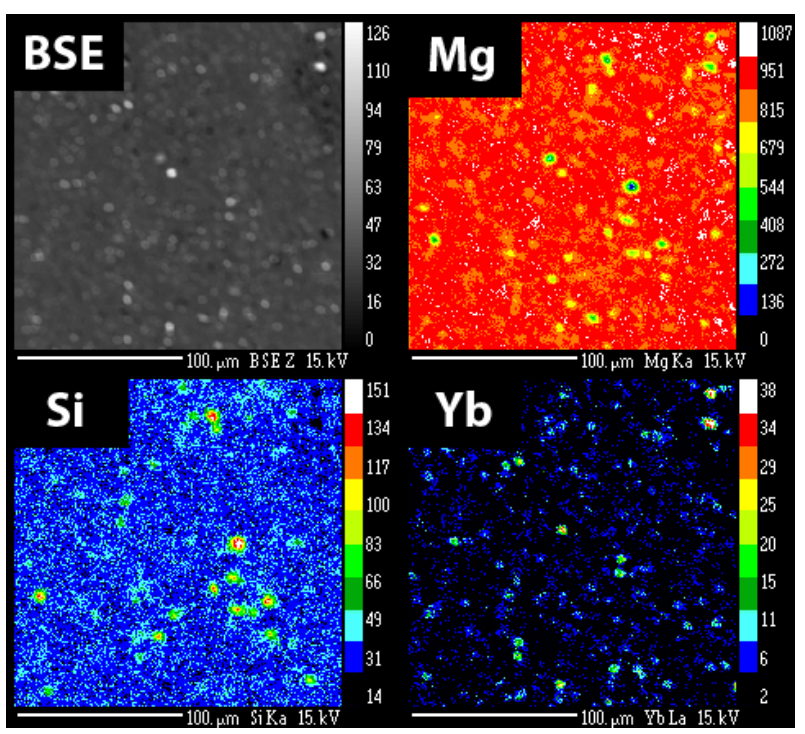

Figure 1: Back scattering electron image (top left) and elemental maps of $\mathrm{Mg}$ (top right), $\mathrm{Si}$ (bottom left) and $\mathrm{Yb}$ (bottom right) of a $1 \% \mathrm{Yb}$ doped $\mathrm{Mg}_{2} \mathrm{Si}$ sample (I).

In addition scanning transmission electron microscopy (STEM) was applied to image both the $\mathrm{Yb}_{3} \mathrm{Si}_{5}$ inclusions and the microstructure of the $1 \% \mathrm{Yb}$ containing sample (I). Atomic number Z-contrast image, an incoherent imaging that is sensitive to the atomic number, can be obtained by collecting the diffracted electrons in the dark field using a high angle annular dark field (HAADF) detector. Energy dispersive X-ray spectroscopy (EDX) analysis can be performed alongside the Z-contrast imaging. Figure 2 (top) shows a HAADF imaging of the $\mathrm{Yb}_{3} \mathrm{Si}_{5}$ inclusion embedded in the $\mathrm{Mg}_{2} \mathrm{Si}$ matrix. The inset image shows the whole $\mathrm{Mg}_{2} \mathrm{Si}$ particle where the magnified image was acquired. The particles with a bright contrast were identified as the $\mathrm{Yb}_{3} \mathrm{Si}_{5}$ phase. The bottom graph shows the energy dispersive $\mathrm{X}$-ray spectroscopy (EDX) data taken from the inclusions to verify the presence of $\mathrm{Yb}$. Atomic 
resolution STEM-HAADF imaging was applied to identify the $\mathrm{Yb}$ distribution at the $\mathrm{Mg}_{2} \mathrm{Si}$ grain boundary. Figure 2a shows the $\mathrm{Yb}$ segregation along the grain boundary of $\mathrm{Mg}_{2} \mathrm{Si}$. Figure $2 \mathrm{~b}$ at higher magnification shows the atomic columns of Si (marked by the red lines) and $\mathrm{Mg}$ (between the solid lines). Since all the Yb dopants with bright contrast fall between the lines one can conclude that $\mathrm{Yb}$ substitutes for $\mathrm{Mg}$ in $\mathrm{Mg}_{2} \mathrm{Si}$ instead of Si. Therefore $\mathrm{Yb}$ plays two roles in $\mathrm{Mg}_{2} \mathrm{Si}$ : it distributes as $\mathrm{Yb}_{3} \mathrm{Si}_{5}$ precipitates and at the grain boundaries substituting for $\mathrm{Mg}$ according to $\mathrm{Mg}_{2-x} \mathrm{Yb}_{x} \mathrm{Si}$. Observations of the formation of $\mathrm{Mg}$ containing phases $\left(\mathrm{Mg}_{3} \mathrm{Sb}_{2}\right.$ and $\left.\mathrm{Mg}_{3} \mathrm{Bi}_{2}\right)$ at the grain boundary have been made by Farahi and coworkers in the investigation of $\mathrm{Sb}$ - and $\mathrm{Bi}$-doped $\mathrm{Mg}_{2} \mathrm{Si}[15]$. 

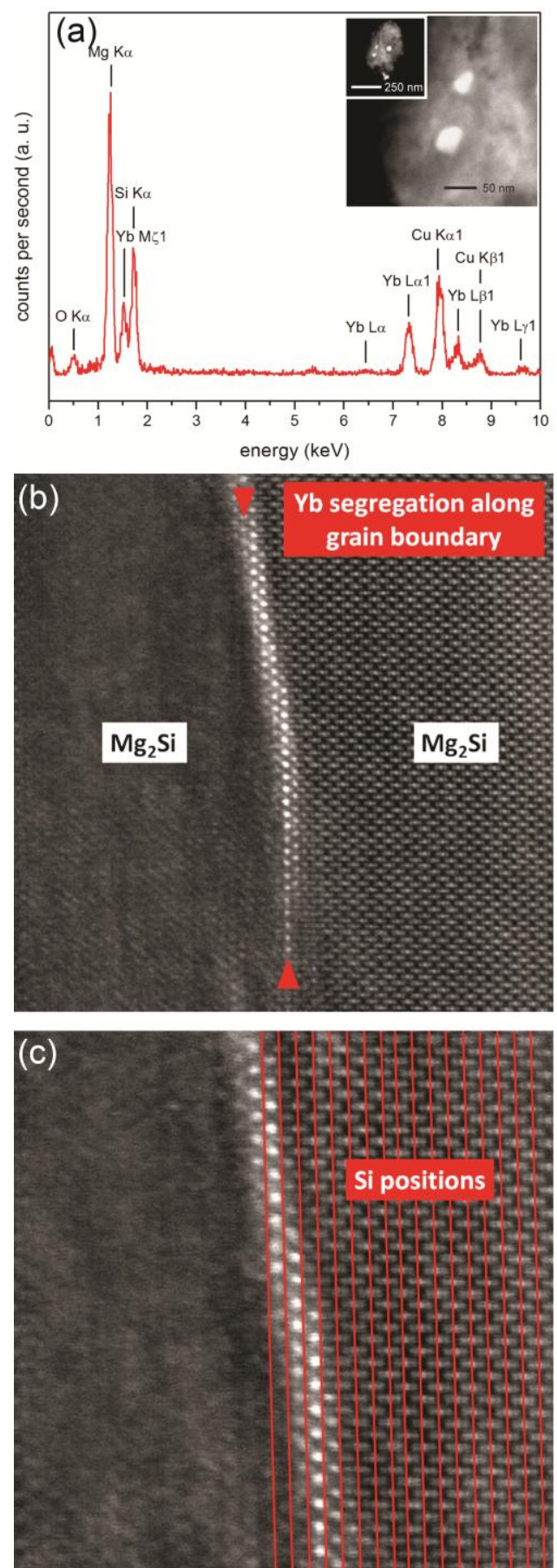

Figure 2: STEM image of a $\mathrm{Mg}_{2} \mathrm{Si}$ particle with $\mathrm{Yb}_{3} \mathrm{Si}_{5}$ inclusions (a). The inclusions have a size of about $40 \mathrm{~nm}$. Inset shows the whole particle of $\mathrm{Mg}_{2} \mathrm{Si}$. EDX was acquired from the same specimen area as shown in the STEM image. The presence of $\mathrm{Yb}$ as well as $\mathrm{Mg}, \mathrm{Si}$ and $\mathrm{O}$ are clearly identified in the EDX spectrum. The $\mathrm{Cu}$ peaks visible are due to the specimen grid used. HAADF-STEM images of a Yb-doped $\mathrm{Mg}_{2} \mathrm{Si}$ sample showing $\mathrm{Yb}$ segregation along the grain boundary (b). The higher magnification image in (c) reveals that the $\mathrm{Yb}$ dopants (bright contrast) do not occupy the Si columns (marked by the line). The Mg columns are located between the lines with a slightly darker contrast than that of $\mathrm{Si}$. The images were high-pass filtered to reduce the high frequency scanning noise. 
The thermal conductivity was calculated from the measured thermal diffusivity $(\alpha)$, the measured geometrical density $(\rho)$ and the specific heat capacity $\left(C_{p}\right)$ using the following equation: $\kappa_{\text {total }}=\mathrm{d} \cdot \mathrm{C}_{\mathrm{p}} \cdot \alpha$. For the specific heat capacity $\mathrm{C}_{\mathrm{p}}$ values published in an earlier report were used [23]. Figure 3 shows the total thermal conductivity of three $\mathrm{Yb}$ doped samples with a nominal $\mathrm{Yb}$ content of $0.2 \%, 0.5 \%$ and $1.0 \% \mathrm{Yb}$ (I-III) in comparison with the thermal conductivity values reported in the literature $[1,16,24]$. The total thermal conductivity of all three samples is lower than plain $\mathrm{Mg}_{2} \mathrm{Si}$, and is also lower than Bi-doped or $\mathrm{Y}$-doped $\mathrm{Mg}_{2} \mathrm{Si}$; thus $\mathrm{Yb}$ doping provides a more significant reduction in thermal conductivity compared to $\mathrm{Y}$ - and Bi-doping. The reduction scales with the amount of $\mathrm{Yb}$, with $1.0 \%$ being the lowest; however, the effect at high temperatures is similar for all samples. Since the electronic contributions to thermal conductivity are larger at the higher temperatures, the lack of influence of $\mathrm{Yb}_{3} \mathrm{Si}_{5}$ precipitates at high temperature is not unexpected. Additionally, since the amount of $\mathrm{Yb}$ substitution for $\mathrm{Mg}$ is very small, it is the increasing amount of $\mathrm{Yb}_{3} \mathrm{Si}_{5}$ that scatters long wavelength phonons more effectively, which dominate heat conduction at lower temperature. At the high temperature regime, short-wavelength phonons are mostly responsible for conduction and since phonon-phonon interactions should dominate, the high temperature thermal conductivity is similar for all of the samples. Therefore when adding Bi to the system, only $0.2 \% \mathrm{Yb}$ was studied (see below). A more detailed image of the thermal conductivity of samples I-III is given in Figure S9 in the Supporting information.

Since $\mathrm{Yb}^{2+}$ is isovalent to $\mathrm{Mg}^{2+}, \mathrm{Yb}$ alloying into the crystal structure of $\mathrm{Mg}_{2} \mathrm{Si}$ should only affect the thermal conductivity of the material and have little to no effect on the carrier concentration and mobility. The formation of $\mathrm{Yb}_{3} \mathrm{Si}_{5}$ inclusions should also lower the thermal conductivity according to Mingos "nano-particle in an alloy" concept [9]. $\mathrm{Yb}_{3} \mathrm{Si}_{5}$ can be considered a Zintl phase, but shows metallic-like behaviour with mixed-valent $\mathrm{Yb}$ [25]. In order to estimate effect of $\mathrm{Yb}$ doping on electrical transport in $\mathrm{Mg}_{2} \mathrm{Si}$, the carrier concentration of the solely $\mathrm{Yb}$ doped samples with low thermal conductivity (I-III) were determined. The results are listed in Table 2. The determined carrier concentrations are in the same range as the ones of undoped $\mathrm{Mg}_{2} \mathrm{Si}$ found in the literature; therefore $\mathrm{Yb}$-doping does not influence the carrier concentrations, as expected. 


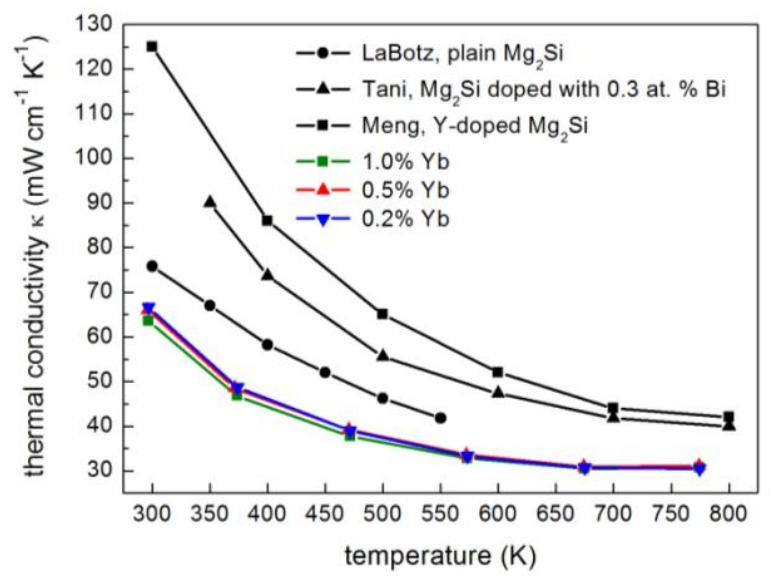

Figure 3: Comparison of the total thermal conductivity of $\mathrm{Mg}_{2} \mathrm{Si}$ from the literature with three $\mathrm{Yb}$-dopant concentrations $(0.2 \%, 0.5 \%$ and $1.0 \%)$ with literature. The literature values were taken from LaBotz [1] for plain $\mathrm{Mg}_{2} \mathrm{Si}$, Tani [16] for optimally doped $\mathrm{Mg}_{2} \mathrm{Si}$ with $\mathrm{Bi}$, and Meng et al. [24] for Y-doped $\mathrm{Mg}_{2} \mathrm{Si}$. 
Table 2: Room temperature charge carrier concentrations of plain $\mathrm{Mg}_{2} \mathrm{Si}$ and $\mathrm{Yb}$ doped $\mathrm{Mg}_{2} \mathrm{Si}$.

\begin{tabular}{lllll}
\hline ID & Yb $(\%)$ & Bi $(\%)$ & synthesis & carrier concentration $\left(\mathrm{cm}^{-3}\right)$ \\
\hline I & 1.0 & - & SPS & $2.1 \times 10^{17}$ \\
II & 0.5 & - & SPS & $7.8 \times 10^{17}$ \\
III & 0.2 & - & SPS & $2.9 \times 10^{17}$ \\
Lit.[26] - & - & solid state reaction & $3.0 \times 10^{16}$ \\
Lit.[26] - & - & mechanical alloying & $2.4 \times 10^{17}$ \\
Lit.[27] - & - & SPS & $4.3 \times 10^{17}$ \\
Lit.[16] - & - & vert. Bridgeman growth & $8.4 \times 10^{17}$ \\
\hline
\end{tabular}

\subsection{Optimization of the Microstructure of $\mathrm{Bi}$ doped $\mathrm{Mg}_{2} \mathrm{Si}$.}

As shown above, the $1 \%$ doping of Yb leads to the lowering thermal conductivity, but does not affect carrier concentration. In order to optimize carrier concentration, the sample with $1 \%$ $\mathrm{Yb}$ and $1 \% \mathrm{Bi}$ dopants were prepared. The $1 \%$ doping with $\mathrm{Bi}$ was reported in the literature as an optimized doping concentration $[10,16,17]$. The samples were prepared by the long ball milling route (Table 1, Experimental) to ensure the homogenous distribution of Bi dopant.

Figure 4 shows the electrical resistivity of the samples (IV-VII, see Table 1 for details) sintered at various temperatures. (Seebeck coefficient data are provided as Supporting Information, SFigure 11). Samples (1\% Yb and 1\% Bi) sintered at higher temperatures, $923 \mathrm{~K}$ and $1023 \mathrm{~K}$ (V and VI, Table 1), showed lower resistivity, with the lowest resistance for sample VII (1\% Bi), prepared by short ball milling.

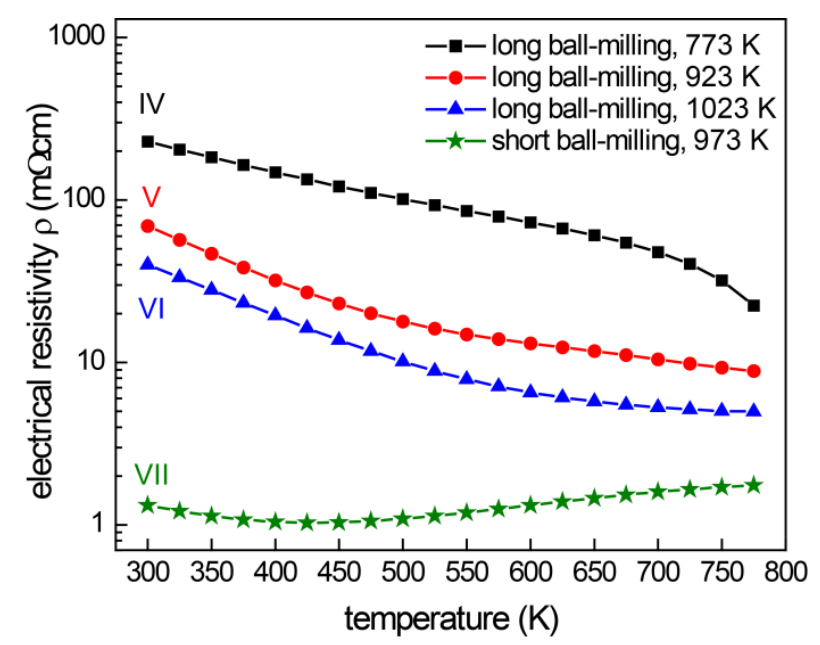

Figure 4: Temperature dependent behaviour of the electrical resistivity $\rho$ for samples $(1 \% \mathrm{Yb}$ and $1 \% \mathrm{Bi}$ ) IV - black, V - red, VI - blue, (1\% Bi) VII - green (See Table 1). The samples 
have been ball milled by different approaches and sintered at different temperature showing significant differences in the electrical resistivity.

In order to check for the effect of sintering temperature on microstructure, and consequently, electrical resistivity, scanning electron microscopy (SEM) images of the microstructure of three samples with $1 \% \mathrm{Yb}$ and $1 \% \mathrm{Bi}$ (IV-VI) were taken (Fig. 5). The samples were cracked and the fracture faces subsequently etched with methanol $(\mathrm{MeOH})$ in order to enable the SEM microstructure investigations. The grain size of the samples is expected to increase with increasing sintering temperature. Sample (IV) (Fig. 5, top) has large grains with sizes up to about $10 \mu \mathrm{m}$ but they are embedded in a matrix of smaller grains with a pronounced size distribution. With larger magnification it becomes clear that some of the smaller grains (sizes of about $0.5-1.5 \mu \mathrm{m}$ ) are also sintered well but again are embedded in a matrix of sub-micron sized particles. In addition, a low degree of small voids are visible which are created during the etching process, as the pellets were fully dense based on geometrical and Archimedes methods. With increasing sintering temperature (samples $\mathrm{V}$ and VI) the grain size increases and the amount of sub-micron sized matrix particles surrounding the crystallites decreases (Fig. 5, middle and Fig. 5, bottom).

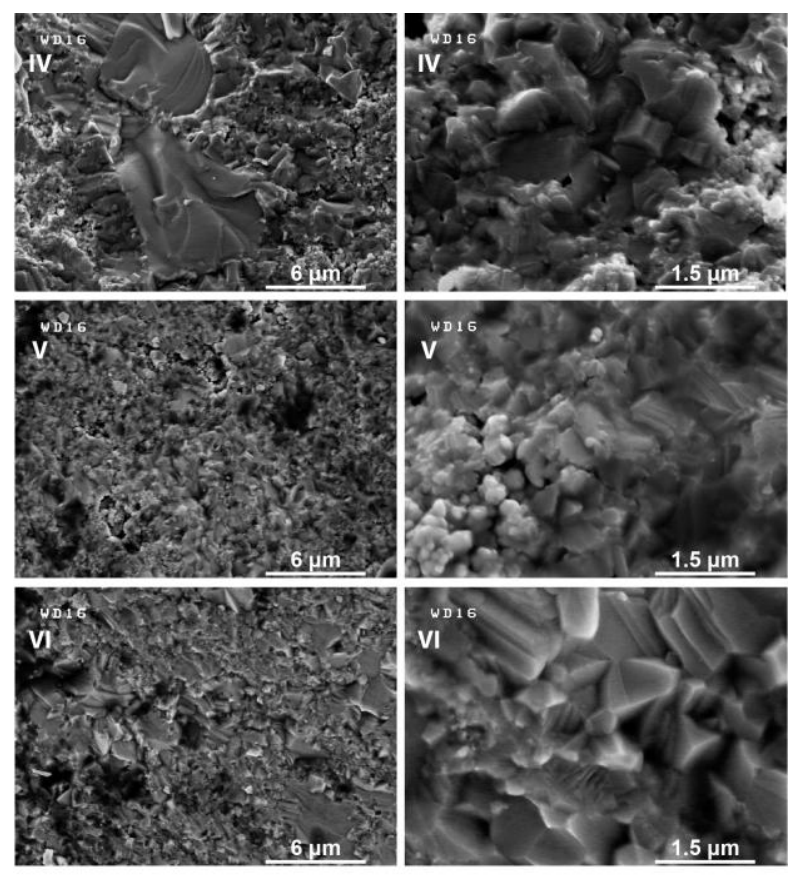

Figure 5: SEM images of the $\mathrm{MeOH}$ etched fracture faces of $1.0 \% \mathrm{Yb}$ doped $\mathrm{Mg}_{2} \mathrm{Si}$ sintered at $773 \mathrm{~K}$ (sample IV, top), $923 \mathrm{~K}$ (sample V, middle) and $1023 \mathrm{~K}$ (sample VI, bottom). The precursor material has been prepared by the long ball milling approach. The images show the microstructure of the sample using $5 \mathrm{k}$ (left) and 20k (right) magnification. 
The microstructure investigation indicates that all three samples are not well sintered (broad grain size distribution), which can be a reason for the high electrical resistivity. As mentioned before, the samples were prepared by the long ball milling method; therefore, severe defects (strain and stress) in the starting material introduced by extended ball milling may negatively impact the microstructure. Additionally, Yb dopant, which was shown to have no effect on carrier concentration, can impact the microstructure by segregating on grain boundaries and impeding the electron transport. Consequently a $1 \%$ Bi doped sample (VII) containing no Yb-dopant was prepared by the short ball milling approach and sintered at 973 K. Short ball milling can be considered as a mixing rather than a milling process, thus fewer defects are expected. The sintering temperature was chosen, because Bux and co-workers state in their work that higher temperatures might lead to significant $\mathrm{Mg}$ loss [17]. The electrical resistivity of this sample (VII) is in the expected range for the optimally doped $\mathrm{Mg}_{2} \mathrm{Si}$ (Figure 4). The microstructure investigation of sample (VII) (green curve in Figure 4) is shown in Figure 6. A homogeneous grain distribution over the whole sample is apparent. The sample exhibits a well sintered microstructure leading to the lowest electrical resistivity as compared to samples (IV-VI) (Figure 6). The resistivity of the sample (VII), is also in line with resistivities reported in the literature for optimal Bi doping [17].

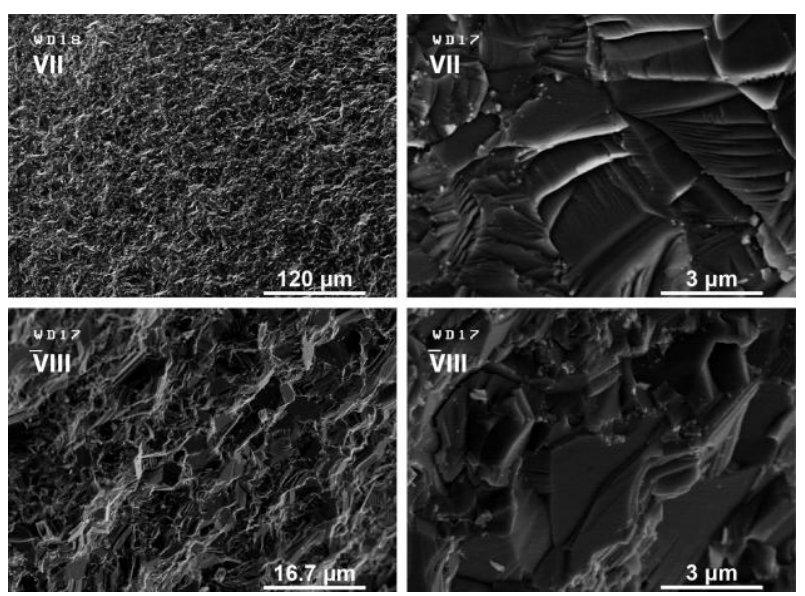

Figure 6: $\mathrm{SEM}$ images of the $\mathrm{MeOH}$ etched fracture faces of $1.0 \% \mathrm{Bi}$ doped $\mathrm{Mg}_{2} \mathrm{Si}$ sintered at $973 \mathrm{~K}$ (sample VII, top) and $1.0 \% \mathrm{Bi}$ and $0.2 \% \mathrm{Yb}$ doped $\mathrm{Mg}_{2} \mathrm{Si}$ sintered at $973 \mathrm{~K}$ (sample VIII, bottom). The precursor material has been prepared by the short ball milling approach. The images show the microstructure of the sample using 250 (left) and 10k (right) magnification.

\subsection{Thermoelectric properties of the $0.2 \% \mathrm{Yb}$ and $1 \% \mathrm{Bi} \mathrm{Mg}_{2} \mathrm{Si}$ sample}


Finally, the thermoelectric properties of the sample co-doped with $\mathrm{Yb}$ and Bi dopants and their impact on the transport properties are discussed. The lowest $\mathrm{Yb}$ concentration of $0.2 \%$ was chosen for sample (VIII) in order to avoid the formation of any possible $\mathrm{Yb}$-Bi phases. $1 \% \mathrm{Bi}$ doping leads to a low electrical resistivity, comparable with other high performance thermoelectric materials. Short ball milling reduces defects in the starting material and sintering temperatures of $973 \mathrm{~K}$ are sufficient to obtain fully dense pellets with a well sintered microstructure. Keeping all these factors in mind, sample (VIII) was prepared by sparkplasma sintering as described before (Table 1). The powder XRD of the sample VIII shows that besides the target $\mathrm{Mg}_{2} \mathrm{Si}$ phase, the sample contains some $\mathrm{MgO}$ and $\mathrm{Yb}_{3} \mathrm{Si}_{5}$ impurity. The presence of $\mathrm{Yb}_{3} \mathrm{Si}_{5}$ is not apparent in the $0.2 \%$ long ball-milled sample. It is probable that the longer ball-milling times are required for the $\mathrm{Yb}$ to replace $\mathrm{Mg}$ in the structure and provide a more finely distributed small grains of $\mathrm{Yb}_{3} \mathrm{Si}_{5}$, not discernable by powder XRD until 1 at.-\%, whereas the short ball-milling time provides large grains of $\mathrm{Yb}_{3} \mathrm{Si}_{5}$ that can be detected by powder XRD. The short ball-milling time results in larger particle size of Si before the SPS reaction, thus affecting the kinetic of the reaction with $\mathrm{YbH}_{2}$ and $\mathrm{MgH}_{2}$ hydrides.

In Figure $7(a)$ the total and lattice thermal conductivity are shown. The thermal conductivity due to conduction of electrons, $\kappa_{\mathrm{e}}$ was estimated using Wiedemann-Franz law: $\kappa_{\mathrm{e}}$ $=\mathrm{LT} / \rho$, where $\mathrm{L}$ is the room temperature Lorenz number, $\mathrm{L}=2.2 \times 10^{-8} \mathrm{~W} \Omega \mathrm{K}^{-2}$, Tabsolute temperature and $\rho$-electrical resistivity. Consistent with the literature, the lattice thermal conductivity, $\kappa_{\text {lattice }}=\kappa_{\text {total }}-\kappa_{\mathrm{e}}$, has a $\sim T^{-1}$ dependence, which indicates that the phonon-phonon interactions are the primary source of the lattice thermal resistance. Overall, the thermal conductivity of sample co-doped with $\mathrm{Yb}$ and $\mathrm{Bi}$ is comparable with literature [1, $10,13,17]$. This is consistent with the interpretation that this sample may not have $\mathrm{Yb}$ replacing $\mathrm{Mg}$ and large grains of $\mathrm{Yb}_{3} \mathrm{Si}_{5}$ that do not contribute as efficiently to the phononphonon interactions.

Figure 7(b) shows the temperature dependent Seebeck coefficient and the electrical resistivity. The Seebeck coefficient has a negative sign, consistent with an $n$-type doped material and increases (absolute value) with increasing temperature, which is typical behaviour for a heavily doped semiconductor. The linear temperature dependent trend of the Seebeck coefficient suggests single parabolic band (SPB) behaviour, while the absolute value is comparable with the literature data [17]. The resistivity of the respective sample (Figure 7(b) shown in red) is slightly temperature dependent initially decreasing with temperature followed by a slight increase at $T>570 \mathrm{~K}$. Similar temperature dependence of $\rho$ has been reported in nanocomposite samples prepared by reactive sintering of $\mathrm{MgH}_{2}$ and $\mathrm{Si}$ [10]. The 
measured carrier concentration amounts to $1 \times 10^{20} \mathrm{~cm}^{3}$ at room temperature, in range for the optimized carrier concentration for thermoelectric performance of $\mathrm{Mg}_{2} \mathrm{Si}[10,17,18]$.
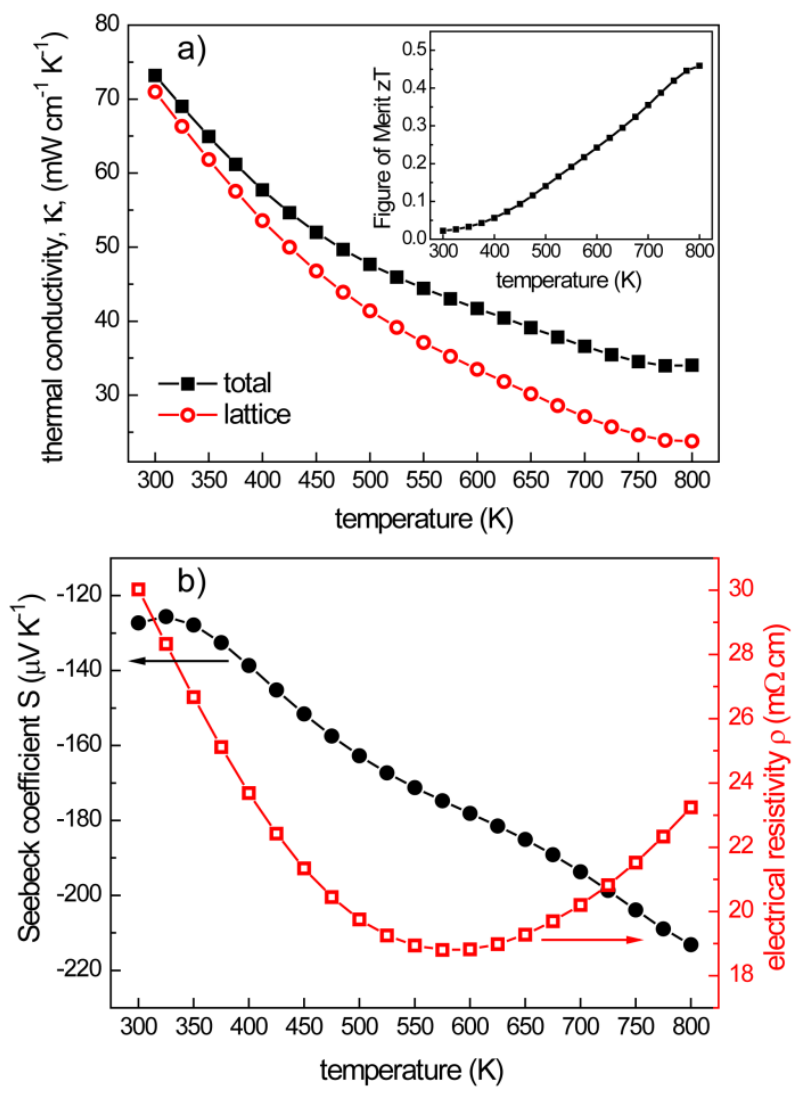

Figure 7: Temperature dependence of the (a) thermal conductivity along with the calculated Figure of Merit zT (inset) and (b) Seebeck coefficient and electrical resistivity for a $0.2 \% \mathrm{Yb}$ and $1.0 \%$ Bi co-doped sample of $\mathrm{Mg}_{2} \mathrm{Si}$ (Sample VIII, Table 1).

In Figure 7(a) (inset) the Figure of Merit $z T$ is shown, calculated from the experimentally determined values. The sample prepared with $0.2 \% \mathrm{Yb}$ and $1.0 \% \mathrm{Bi}$ shows a maximum $z T$ of 0.46 at $800 \mathrm{~K}$. The relatively high absolute value of resistivity of $\sim 25 \mathrm{~m} \Omega \mathrm{cm}$ can be explained by the low carrier mobility, which is most likely caused by ionized impurity scattering or $\mathrm{Yb}$ segregation at the grain boundaries. This effect is responsible for the moderate value of thermoelectric figure of merit. By itself, Yb-doping has a positive effect on the thermal transport and does not alter the carrier concentration. However, further optimization is needed as the segregation of $\mathrm{Yb}$ at the grain boundaries presumably impedes the electron mobility, thus unfavorably increasing electrical resistivity.

\section{Conclusions}

Magnesium silicide $\mathrm{Mg}_{2} \mathrm{Si}$ is a low density, low cost thermoelectric material which requires tuning in order to achieve suitable Figure of Merit $z T$ values. In our investigations we 
focused on samples that were doped with $\mathrm{Yb}$ in order to decrease the thermal conductivity. Excess $\mathrm{Yb}$, beyond the potential solubility limit leads to the formation of one of the various $\mathrm{Yb}_{\mathrm{x}} \mathrm{Si}_{\mathrm{y}}$ phases known in literature [25, 28-33]. With the help of EMPA and STEM structure characterization the $\mathrm{Yb}$ dopant is found to be localized in the prepared materials: both the formation of ytterbium silicide $\mathrm{Yb}_{3} \mathrm{Si}_{5}$ inclusions within the $\mathrm{Mg}_{2} \mathrm{Si}$ matrix and segregation of $\mathrm{Yb}$ in the grain boundary with $\mathrm{Yb}$ substitution of $\mathrm{Mg}$ observed. We have investigated the influence of the microstructure on the transport properties of $\mathrm{Mg}_{2} \mathrm{Si}$ prepared from $\mathrm{MgH}_{2}$ and $\mathrm{Si}$ in a one-step fabrication process by spark plasma sintering. It is shown that the sintering and ball milling conditions have a significant influence on the microstructure of the final material and consequently on the transport properties. The sample with a co-doping of $0.2 \%$ $\mathrm{Yb}$ and $1.0 \% \mathrm{Bi}$, exhibits a $z T$ of 0.46 at $800 \mathrm{~K}$, which is slightly lower what is reported for the best results from $\mathrm{Bi}$ doping in the literature [18]; however, with the understanding gained from the microstructure and the influence of co-doping of the system, further optimization of the $\mathrm{Mg}_{2} \mathrm{Si}$ system and additional increases in $z T$ should be possible.

\section{Experimental}

\subsection{Synthesis}

$\mathrm{Mg}_{2} \mathrm{Si}$ samples doped with $\mathrm{Yb}$ were prepared using $\mathrm{MgH}_{2}$ (Alfa Aesar, powder, $98 \%$ ), $\mathrm{Si}$ (Aldrich, powder 325 mesh, $99 \%$ ), and $\mathrm{Yb}$ powder (American Elements, 5N). The $\mathrm{Yb}$ powder was determined to be $\mathrm{YbH}_{2}$ by powder $\mathrm{X}$-ray diffraction and used without further purification. To adjust the carrier concentration, Bi (Johnson Matthey Electronics, rod, $99.9999 \%$ ) was added to produce n-type doped materials. All starting materials (total mass of about $12 \mathrm{~g}$ ) were loaded into a tungsten carbide-lined vial with additional O-ring seals in an argon filled glove box. The vial was sealed in a Ziploc bag for an additional oxidation protection for the material during the ball milling process. For the long ball milling, all starting materials were added and ball milled for 9 hours in 15 minute on/off intervals using two large tungsten carbide balls. For the short ball milling, $\mathrm{Si}$ and $\mathrm{Bi}$ were continuously shaken for 30 minutes. The ball mill was transferred into the glove box and $\mathrm{MgH}_{2}$ and $\mathrm{YbH}_{2}$ were added, sealed as described above, and the mixture was ball milled for 30 minutes. At the end of the ball milling process, the vial was transferred back into the glove box and stored there. The long ball milling process can be considered to be an effective milling step, including particle reduction, while the short ball milling process is a mixing rather than milling step. The starting material was reacted and compacted in a one-step reaction using spark-plasma-sintering techniques with the following temperature and pressure profile. The 
dopants are provided as at.-\%. The SPS experiments were carried out on a Dr. Sinter SPS825S apparatus (SPS Syntex Inc., Kanagawa, Japan) using graphite dies with a diameter inner diameter $12.7 \mathrm{~mm}$ and a sample load of approximately $0.5 \mathrm{~g}$. The temperatures given in Table 1 were measured from the outside of the die with a K-type thermocouple. The dies were aligned in the instrument and dynamic vacuum was applied to the sample chamber. The sample was kept under an axial compressive pressure of $50 \mathrm{MPa}$. After achieving an acceptable vacuum, the temperature ramped to $623 \mathrm{~K}$ within 3 minutes, and then slowly to $723 \mathrm{~K}$ within 10 minutes. Since sintering was carried out under dynamic vacuum and the reaction onset was indicated by an increase in pressure caused by the hydrogen released during the reaction. After the reaction ended the pressure was raised to $115 \mathrm{MPa}$ within 2 minutes, while the sample was heated in $10 \mathrm{~min}$ to $10 \mathrm{~K}$ below the final sintering temperature given in Table 1. The final sintering temperature was reached within 1 minute followed by a dwelling step for 10 minutes before the sample was slowly cooled down to $673 \mathrm{~K}$ within 10 minutes. The applied pressure was reduced once the cooling started. An optical image of a pellet obtained by this technique is given in Figure 8 .

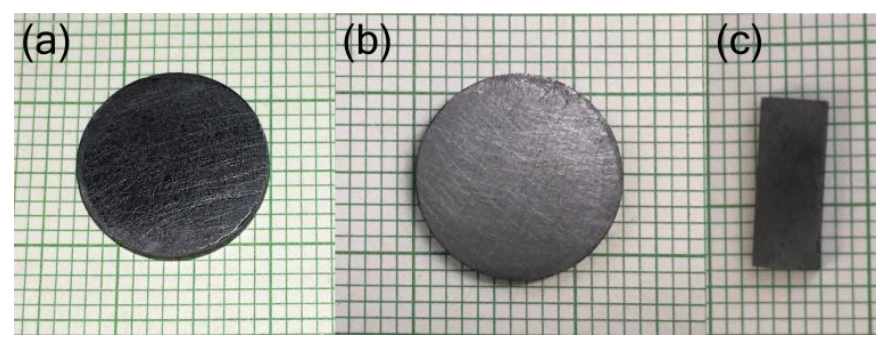

Figure 8: Optical images of a $12.7 \mathrm{~mm}$ pellet of $\mathrm{Mg}_{2} \mathrm{Si}$ obtained via spark plasma sintering viewed on a mm grid and photographed at an angle (a) and from on top (b). (c) Bar cut from the pellet for sample VIII which was used for transport property measurements.

\subsection{Thermal Analysis}

TG/DSC measurements were carried out on a Netzsch STA 409C instrument. Typically 20 $\mathrm{mg}$ of the starting material was pressed into a small pellet of $2 \mathrm{~mm}$ diameter and transferred into $\mathrm{Al}_{2} \mathrm{O}_{3}$ crucibles with lid in an argon filled drybox. The samples were transferred into the TG/DSC instrument and heated under a constant Argon stream to $873 \mathrm{~K}$ with a heating rate of $10 \mathrm{~K} \mathrm{~min}^{-1}$. Since $\mathrm{H}_{2}$ is formed during the reaction, the TG/DSC measurement was only heated till $873 \mathrm{~K}$ to protect the platinum parts of the instrument.

\subsection{X-ray Powder Diffraction}


X-ray powder diffraction data were collected with a Bruker D8 Advance diffractometer using $\mathrm{Cu} K \alpha$ radiation $(40 \mathrm{KeV}, 40 \mathrm{~mA})$ on powders prepared from pulverizing the sintered pellets. Data were collected on a rotating sample $(15 \mathrm{rpm})$ from $10^{\circ}$ to $90^{\circ}(2 \Theta)$, with a step size of $0.01944^{\circ}$ and a step time of $1.25 \mathrm{~s}$. Data acquisition was performed using the Bruker Command Center software. Lattice parameter refinements for all present phases as well as full pattern fitting was conducted with the FullProf Suite [34].

\subsection{Electron Microprobe Analysis}

Fragments of the pressed pellet shaped samples were mounted in epoxy and polished before they were characterized using a Cameca SX-100 electron microprobe equipped with five wavelength dispersive spectrometers. The instrument was operated at $10 \mathrm{kV}$ accelerating voltage and $10 \mathrm{nA}$ current with a beam diameter of approximately $1 \mu \mathrm{m}$. Elemental mapping was used to determine the homogeneity of the samples over a region of at least $200 \times 200 \mu \mathrm{m}^{2}$. $\mathrm{Yb}, \mathrm{Si}$ and $\mathrm{MgO}$ were used as standards for all samples.

\subsection{Thermoelectric measurements}

Electrical resistivity and Seebeck coefficient were measured on a Linseis LSR3 instrument. The samples were measured in helium atmosphere with ultra-high purity (99.999\%). Thermal diffusivity was measured using a Netzsch laser flash diffusivity system from room temperature to $775 \mathrm{~K}$. The thermal conductivity was calculated using the relation $\kappa=\alpha \cdot \mathrm{C}_{\mathrm{p}}$. $\mathrm{d}$, where $\alpha$ is the measured thermal diffusivity, $\mathrm{C}_{\mathrm{p}}$ is the specific heat capacity taken from the literature [23] and $\mathrm{d}$ is the measured geometric density. The carrier concentration was determined under high vacuum by custom made set-ups described elsewhere [35]. Hall effect of sample VIII was measured by a five-wire measurement on Quantum Design Physical Property Measurement System (PPMS) in the temperature range of $200 \mathrm{~K}-400 \mathrm{~K}$ and magnetic field up to 5 T. Carrier concentration (n) and electron mobility $(\mu)$ were calculated form Hall effect and resistivity $(\rho)$ according to equation: $\rho^{-1}=n e \mu$.

\subsection{Scanning Electron Microscopy}

Scanning electron microscopy was conducted on a Hitachi S-4100T Hitachi HTA America FE-SEM with an attached Oxford INCA Energy EDS unit.

\subsection{Scanning Transmission Electron Microscopy}


The electron transparent specimen was prepared by mechanical grinding and polishing using a pellet of $\mathrm{Mg}_{2} \mathrm{Si}$ with $1.0 \% \mathrm{Yb}$ doping. Atomic resolution STEM-HAADF images of the $\mathrm{Yb}$ segregation inside the GB were obtained using the Cs-aberration corrected JEOL2100F microscope operated at $200 \mathrm{kV}$, with a HAADF detector collection angle of 70190 mrads. The STEM-HAADF images and the EDX spectrum of the $\mathrm{Yb}_{3} \mathrm{Si}_{5}$ inclusions were acquired using an aberration corrected FEI Titan at 300kV in PNNL.

\section{Acknowledgements}

We thank Greg Baxter for sample preparation for microprobe analysis and Nick Botto for the measurements. We gratefully acknowledge financial support from NSF/DOE Partnership CBET-1048799, DMR-1405973, and UC Davis.

A portion of this research is part of the Chemical Imaging Initiative conducted under the Laboratory Directed Research and Development (LDRD) Program at Pacific Northwest National Laboratory (PNNL) and was performed at the Environmental Molecular Sciences Laboratory (EMSL), a national scientific user facility sponsored by the Department of Energy's Office of Biological and Environmental Research (DOE BER) located at PNNL, a multiprogram national laboratory operated by Battelle Memorial Institute for the DOE under Contract DE-AC05-76RL01830.

Part of this work was carried out at the Jet Propulsion Laboratory, California Institute of Technology, under a contract with the National Aeronautics and Space Administration. This work supported by the NASA Science Missions Directorate's Radioisotope Power Systems Thermoelectric Technology Development Project. 


\section{References}

[1] R.J. LaBotz, D.R. Mason, Journal of the Electrochemical Society 110 (1963) 121-126.

[2] E.N. Nikitin, V.G. Bazanov, V.I. Tarasov, Soviet Physics - Solid State 3 (1962) 2648-2651.

[3] Y. Noda, H. Kon, Y. Furukawa, I.A. Nishida, K. Masumoto, Materials Transactions 33 (1992) 851-855.

[4] Y. Noda, H. Kon, Y. Furukawa, N. Otsuka, I.A. Nishida, K. Masumoto, Materials Transactions 33 (1992) 845-850.

[5] Q.S. Meng, L.Q. Wang, B.S. Li, L.Z. Ding, S.P. Chen, Advanced Materials Research 79-82 (2009) 1639-16421642.

[6] Y. Niwa, Y. Todaka, M. Umemoto, K. Yamana, K. Kinoshita, M. Tanizawa, Journal of the Japan Institute of Metals 72 (2008) 693-697.

[7] Q. Zhang, X.B. Zhao, H. Yin, T.J. Zhu, Journal of Alloys and Compounds 464 (2008) 9-12.

[8] S. Chen, X. Zhang, W. Fan, T. Yi, D.V. Quach, S. Bux, Q. Meng, S.M. Kauzlarich, Z.A. Munir, Journal of Alloys and Compounds 625 (2015) 251-257.

[9] N. Mingo, D. Hauser, N.P. Kobayashi, M. Plissonnier, A. Shakouri, Nano Letters 9 (2009) 711715.

[10] T. Yi, S. Chen, S. Li, H. Yang, S. Bux, Z. Bian, N.A. Katcho, A. Shakouri, N. Mingo, J.-P. Fleurial, N.D. Browning, S.M. Kauzlarich, Journal of Materials Chemistry 22 (2012) 24805-24813.

[11] S. Wang, N. Mingo, Applied Physics Letters 94 (2009) 203109-3.

[12] J. Tani, H. Kido, Physica B: Condensed Matter 364 (2005) 218-224.

[13] J. Tani, H. Kido, Intermetallics 15 (2007) 1202-1207.

[14] Q. Zhang, X. Su, Y. Yan, H. Xie, T. Liang, Y. You, X. Tang, C. Uher, ACS Appl Mater Interfaces 8 (2016) 3268-76.

[15] N. Farahi, M. VanZant, J. Zhao, J.S. Tse, S. Prabhudev, G.A. Botton, J.R. Salvador, F. Borondics, Z. Liu, H. Kleinke, Dalton Trans 43 (2014) 14983-91.

[16] J.-i. Tani, H. Kido, Physica B: Condensed Matter 364 (2005) 218-224.

[17] S.K. Bux, M.T. Yeung, E.S. Toberer, G.J. Snyder, R.B. Kaner, J.-P. Fleurial, Journal of Materials Chemistry 21 (2011) 12259-12266.

[18] N. Farahi, M. VanZant, J. Zhao, J.S. Tse, S. Prabhudev, G.A. Botton, J.R. Salvador, F. Borondics, Z. Liu, H. Kleinke, Dalton Transactions 43 (2014) 14983-14991.

[19] S. Fiameni, S. Battiston, S. Boldrini, A. Famengo, F. Agresti, S. Barison, M. Fabrizio, Journal of Solid State Chemistry 193 (2012) 142-146.

[20] E. Savary, F. Gascoin, S. Marinel, R. Heuguet, Powder Technology 228 (2012) 295-300.

[21] J. Schmidt, R. Niewa, M. Schmidt, Y. Grin, Journal of the American Ceramic Society 88 (2005) 1870-1874.

[22] A.-L. Chaudhary, D.A. Sheppard, M. Paskevicius, C. Pistidda, M. Dornheim, C.E. Buckley, Acta Materialia 95 (2015) 244-253.

[23] M. Riffel, J. Schilz, XVI International Conference on Thermoelectrics, ICT, Proceedings 1997, 1997, pp. 283-286.

[24] Q.S. Meng, W.H. Fan, R.X. Chen, Z.A. Munir, Journal of Alloys and Compounds 509 (2011) 7922-7926.

[25] R. Pöttgen, R.D. Hoffmann, D. Kußmann, Zeitschrift für Anorganische und Allgemeine Chemie 624 (1998) 945-951.

[26] H.J. Lee, Y.R. Cho, I.-H. Kim, Journal of Ceramic Processing Research 12 (2011) 16-20.

[27] M. Akasaka, T. lida, A. Matsumoto, K. Yamanaka, Y. Takanashi, T. Imai, N. Hamada, Journal of Applied Physics 104 (2008) 013703-8.

[28] F. Canepa, S. Cirafici, F. Merlo, A. Palenzona, Journal of Magnetism and Magnetic Materials 118 (1993) 182-186.

[29] E.I. Gladyshevskii, P.I. Kripyakevich, Journal of Structural Chemistry 5 (1965) 789-794.

[30] A. landelli, A. Palenzona, G.L. Olcese, J. Less-Com. Met. 64 (1979) 213-220.

[31] C. Kubata, F. Krumeich, M. Wörle, R. Nesper, Zeitschrift für Anorganische und Allgemeine Chemie 631 (2005) 546-555.

[32] J.A. Perri, I. Binder, B. Post, Journal of Physical Chemistry 63 (1959) 616-619. 
[33] A. Grytsiv, D. Kaczorowski, A. Leithe-Jasper, V.H. Tran, A. Pikul, P. Rogl, M. Potel, H. Noël, M. Bohn, T. Velikanova, Journal of Solid State Chemistry 163 (2002) 178-185.

[34] J. Rodríguez-Carvajal, Physica B: Condensed Matter 192 (1993) 55-69.

[35] S.K. Bux, R.G. Blair, P.K. Gogna, H. Lee, G. Chen, M.S. Dresselhaus, R.B. Kaner, J.-P. Fleurial, Advanced Functional Materials 19 (2009) 2445-2452. 